Editorial Traditional Chinese medicine (TCM) is a great cultural heritage of China from generation to generation with a history of thousands of years. TCM with its rich clinical experiences and impressive efficacy is quite different from modern western medicine neither in the therapeutic philosophy or the principles. It is a pity to human health maintenance that TCM has not been yet easily comprehensible by the medical and academic circles worldwide. Based on years of studies on Chinese herbal medicine, biomechanics and pharmacology, the authors introduce their research progress and gains in the borderline subjects, especially the new concept of "biomechanopharmacology". We believe the article opens a new approach for TCM prevention and treatment, particularly for cardiovascular and cerebrovascular diseases. We hope that the article will arouse attention and discussions among the readers. It is our expectation that scientists will put more concerns and efforts in TCM researches. TCM will be internationally accepted as one of the main medical means for health maintenance when it is gained a sound basis of modern scientific annotations.

\title{
Chinese medicine, flowing blood, biomechanopharmacology and future preventive medicine
}

\author{
HAN Dong $^{1 *} \&$ LIAO FuLong ${ }^{1,2 *}$ \\ ${ }^{1}$ National Center for Nanoscience and Technology, Beijing 100190, China; \\ ${ }^{2}$ China Academy of Chinese Medical Sciences, Beijing 100700, China
}

Received December 21, 2011; accepted April 23, 2012

\begin{abstract}
Activating Blood Circulation to Remove Blood Stasis (ABCRBS) has long been the most fundamental therapeutic principle since the age of Yellow Emperor (circa 2600 BC) in Chinese medicine. Based on bio-responses of blood shear stress, which was not considered in Chinese medicine, we have initiated the merging of biomechanics with pharmacology and Chinese medicine, establishing the new discipline-biomechanopharmacology. Practice of exercise is a simple and self-regulatory way to promote shear stress in the circulation. Interestingly, acupuncture and massage are also linked with shear stress regulation. These procedures are expected to maintain normal functions of endothelial cells (ECs) by regulating shear stress levels of flowing blood. The statement of "Diseases are prevented as blood flow is promoted" by the renowned ancient physician Huatuo, who presented the wise foresight of the efficacy of shear stress, would likely begin a new chapter in preventive pharmacology and medicine in the years to come.
\end{abstract}

Chinese medicine, Activating Blood Circulation to Remove Blood Stasis, flowing blood, biomechanopharmacology, preventive medicine

Citation: Han D, Liao F L. Chinese medicine, flowing blood, biomechanopharmacology and future preventive medicine. Chin Sci Bull, 2012, 57: 3541-3546, doi: $10.1007 / \mathrm{s} 11434-012-5226-8$

Science magazine once asked a puzzling question: "How much can human life span be extended?" [1]. According to life-span extension in species, scientists have been convinced that humans will routinely coast beyond 100 or 110

*Corresponding authors (email: dhan@nanoctr.cn, flliao@nanoctr.cn) years of age. If that happens, the vascular disorders that occur during the delayed body retrogression, with abnormal interactions among blood, blood flowing and blood vessels, will become an even greater challenge. Herbal medicine, acupuncture, massage, exercise and life styles have contributed a great deal to the healthcare of people in China for 
thousands of years. Chinese medicine is therefore expected to be one of the potential means in healthcare to resolve the vascular problems in the extended life span.

\section{Circulation, hemorheology and Chinese medicine}

Regarding the circulatory system, the pioneer of biorheology A. L. Copley pointed out the need to focus on hemorheology, a multidisciplinary field on flow and deformation of blood and blood vessels at macroscopic, cellular and even molecular levels [2]. The marvelous interactions among blood, blood vessel and blood flow maintain the physiological functioning of endothelium. Once the balance is disturbed, it is highly likely that vascular diseases and the successive disorders may follow [3]. In the arterial system, disturbed flow pattern in branch points and curvatures not only causes the preferential localization of atherosclerotic lesions, but also results in postsurgical neointimal hyperplasia, contributing to pathophysiology of clinical conditions such as in-stent restenosis, vein bypass graft failure, and transplant vasculopathy and aortic valve calcification. In the venous system, disturbed flow resulting from reflux, outflow obstruction, and/or stasis leads to venous inflammation and thrombosis, and hence the development of chronic venous diseases [4].

Recent international biomedical research has demonstrated that the maintenance of physiological laminar shear stress (SS) due to blood flow is crucial for normal vascular functions, including the regulation of vascular diameterthe most influential factor in blood flow and volume regulation-and the inhibition of proliferation, thrombosis and inflammation of the vessel wall [4,5]. Furthermore, SS together with chemical stimuli, such as VEGF, may regulate intracellular signaling pathways. These results highlight the complexity of cell signaling transduction in response to the following blood [6-8].

In Chinese medicine, the concept of "Xue" (blood) is similar to the modern understanding of flowing blood. The healthy blood circulation is described as "blood going", while the state of "blood stasis" is considered as a main pathological syndrome (Xue Yu syndrome) in many disease progressions. In view of hemorheology, insufficient SS is an underlying characteristic of blood stasis syndrome. The statement of "Diseases are prevented as blood flow is promoted" by the renowned ancient surgeon Huatuo (145-208 $\mathrm{AD}$ ) is interestingly a wise foresight of the efficacy of shear stress.

In fact, ABCRBS, one of the main therapeutic principles in Chinese medicine, has been explored since the time of Yellow Emperor (circa 2600 BC). So far, herbs in the ABCRBS category account for over $10 \%$ of herbal medicine in the modern Chinese Pharmacopoeia. In the integrated approach combining traditional Chinese and western medicine, blood stasis syndrome is diagnosed by integrated orthodoxical and laboratory criteria, including hemorheological and microcirculatory parameters. References in the literatures show that the diagnostic value of multi-hemorheological parameters is significant [9]. Blood flowing and its interaction with the vessel are one of the central concerns, which will be the first candidate to bridge the gap between Chinese and modern medicine.

\section{ABCRBS herb and biomechanopharmacology}

With regard to healthcare using Chinese herbal medicine, herbs of ABCRBS are an issue in ancient practice that deserves modern investigation. The heritages unearthed from Mawangdui Han Tomb include some herbs of ABCRBS. Tetramethylparazine (TMP, see Figure 1), contained in Rhizoma Ligustici chuanxiong, and is a popular herb of ABCRBS, has been used as an inhibitor of platelet aggregation in China since the 1970s. Recently, Chinese pharmacologists have been interested in its effects on the interaction between drug-contained flowing blood and ECs because the in vivo life-long SS with various patterns and its biological effects are undoubtedly present whenever a medicine is introduced into the blood. There is always a joint effect of SS and the pharmacological factor (drug). The joint effect of TMP and SS on the early apoptosis of cultured rat cerebral microvascular ECs (rCMECs) was investigated by the administration of TMP at different levels of SS generated by a rotational cone-plate rheometer. Interestingly, the medium-dose combinations of SS and TMP, rather than the high-dose combinations, appear to be more effective at lowering the apoptosis of rCMECs [10]. This indicates that the classical pattern of pharmacological effects could be significantly modified when SS as a biomechanical factor of influencing endothelial function is involved. In general, the effectiveness of a medicine might be significantly modified when mechanical factors are also present. As a consequence of merging the biomechanical factors with the pharmacological factors, joint investigation of the two factors has become a research basis.

A recent study reported that the combination of Sal B and SS treatment dramatically inhibited TNF-alpha-induced NF-kappaB activation, as evidenced by IkappaB-alpha degradation and p65 nuclear translocation in human aortic ECs. This provides the first biomechanopharmacological evidence that Sal B has a combination effect with SS to reduce the expression of three adhesion molecules (VCAM-1, ICAM-1 and E-selectin), leading to reduced monocyte adhesion to ECs by inhibiting the NF-kappaB signaling pathway [11]. SS is not always beneficial for the interactions among blood, blood flow and blood vessel. Shear-induced platelet aggregation (SIPA) under high shear stress in regions of stenosis attracted attention due to its involvement in vascular diseases, especially in life-threatening thrombosis 


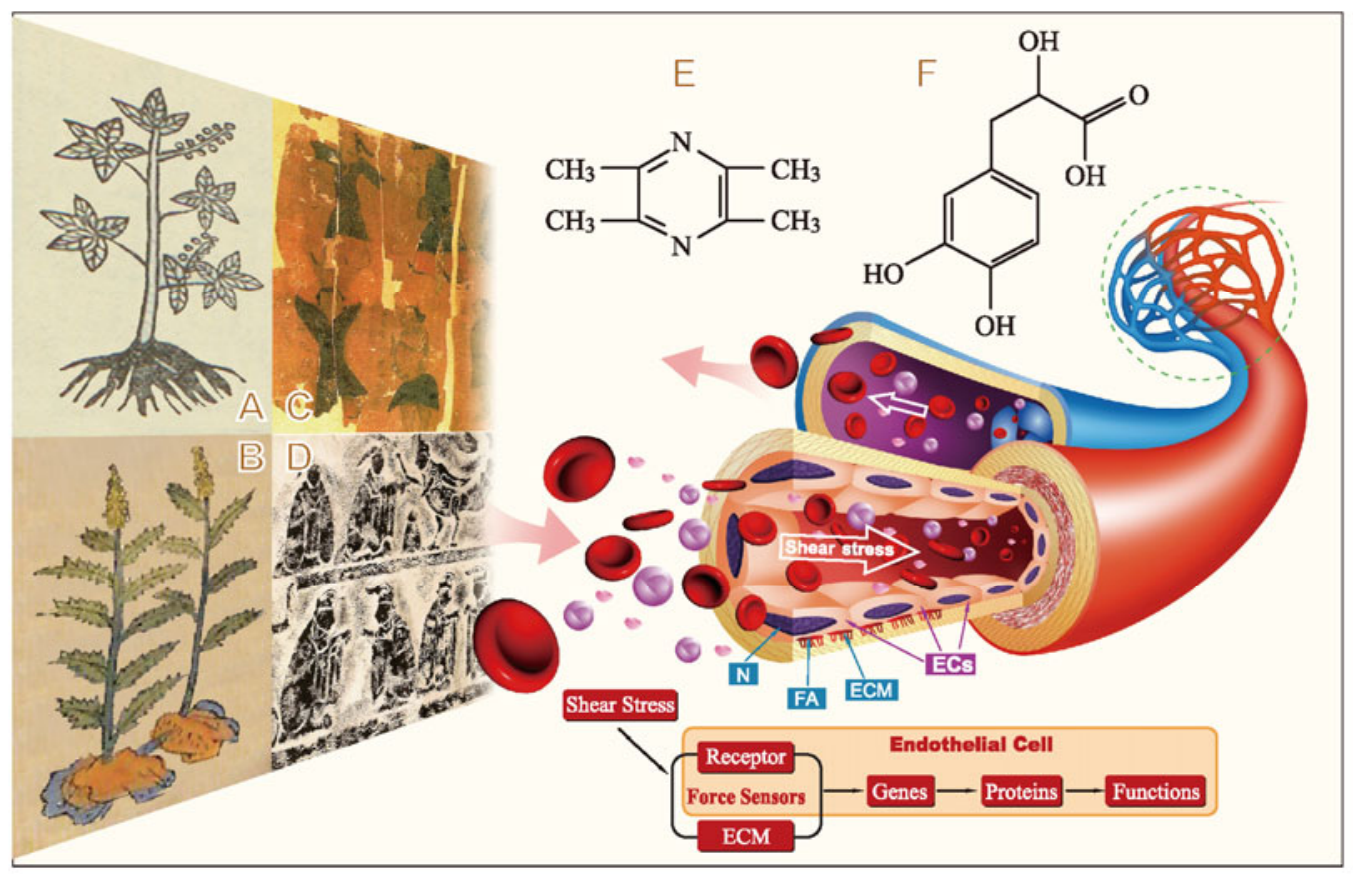

Figure 1 The integrated concepts in the article. The right figure illustrates an artery with its inner lining of ECs connected to a venous vessel by microvasculature. The flowing blood exerts shear stress onto the EC surface. The force signal received by the EC induces a series of changes at the levels of gene and protein expressions, and finally functional regulation. The left figure introduces briefly the traditional Chinese medicine background. (A) and (B) show two typical herbs of ABCRBS, Salvia miltiorrhiza and Rhizoma Ligustici chuanxiong, respectively, from ancient herbal literatures. The two molecular structures are TMP (E) contained in Rhizoma Ligustici chuanxiong, and DLA (F) contained in Salvia miltiorrhiza. They have been extensively investigated pre-clinically and applied clinically, and both have biomechanopharmacological effects. Drawing of physical and breathing exercise on silk (C) unearthed from a Han tomb is an early archaeological evidence of exercise employed in healthcare in China more than two thousand years ago. The brick sculpture (D) of Han dynasty illustrates that the well-known physician in Archaic China, Bian Que, was practicing acupuncture on patients. Exercise, herbal medicine and acupuncture are all involved in the intervention of blood flow and hence shear stress, thus regulating the endothelial functions. N: Cell nucleus. FA: Focal adhesion. ECM: Extracellular matrix. ECs: Endothelial cells.

[12]. SIPA can result from the activation of calcium channel of platelet membrane as a result of the interaction between plasma vWF and membrane GP Ib. SIPA has undesirable implications because conventional anti-platelet drugs such as aspirin are of little use. The development of inhibitors of SIPA has been a pharmaceutical target since the beginning of 1990s [13]. Extracts from Chinese herbal medicine showed actual efficacy on inhibiting SIPA [14]. TMP and diallyl trisulfide extracted from Bulbus Allium have been reported to inhibit SIPA in a dose-dependent manner [15], and the combined application of TMP and SAB has additive effects on inhibiting SIPA [16]. In other cases, the simultaneous applications of peroxidation-induced factors and mechanical force on cultured rCMECs with morphological tight junction, can cause a breakdown of cytoskeleton fiber networks in some regions of these cells and lead to a damage of the tight junctions between them. In addition, it appears likely that the applied mechanical force can directly disrupt the filament network [17]. In ischemia/reperfusion in vivo, the mechanical force acting on the cytoskeleton fibers in microvasular ECs may increase remarkably because of the neutrophil accumulation/adhesion/rolling, blood hyperviscosity, and/or increase in membrane protrusions. It might be possible that the mechanical forces from reperfusion after ischemia would injure ECs and cause damage of the blood-brain barrier. This kind of injury from mechanical forces has not been the focus of clinical medicine thus far.

Salvia miltiorrhiza root, another popular ABCRBS herb, has long been used in Chinese medicine for treatment of various diseases involving microcirculatory disturbances. This herbal drug contains many active water-soluble compounds, including protocatechuic aldehyde (PAl), 3, 4dihydroxyphenyl lactic acid (DLA, see Figure 1), and salvianolic acid B (Sal B). These compounds, as well as the water-soluble fraction of Salvia miltiorrhiza root, have the abilities to scavenge peroxides and to inhibit the expression of adhesion molecules in vascular endothelium and leukocytes. The ameliorating effect of compounds derived from Salvia miltiorrhiza root on microcirculatory disturbance and target organ injury after ischemia/reperfusion has been reviewed [18].

The herbs of ABCRBS have the common function of activating blood circulation. There have been numerous clinical and experimental reports on the improvement of blood circulation achieved by herbs of ABCRBS since the 1980's [19]. Thus, the SS is maintained at appropriate higher levels with the application of herbal intervention in accordance 
with the pathological low levels. Then, the beneficial bio-responses of SS can be expected. Recently, the active ingredients of ABCRBS herbs have been re-composed to form new medicines. Xiongshao Capsule is one of them, consisting of active ingredients from Rhizoma Ligustici chuanxiong and Radix Paeonia lactiflora. The capsule significantly reduced the late lumen narrowing resulting from vascular remodelling in the porcine coronary injury model [20-22]. The capsule has been applied to minimize restenosis after percutaneous transluminal coronary angioplasty with marked benefit.

In fact, flowing blood is more than a transporter and deliverer of oxygen, nutrition, metabolic products and drugs. Its biomechanical impact on various functions of ECs should be fully addressed. According to the principle of pharmacology that efficacy is the therapy goal of drug, SS may be regarded as a multi-targeted drug since potential multi-efficacy is gained by regulating SS at different levels. As a result, we initiated the merging of biomechanics, pharmacology and Chinese medicine, establishing the new discipline-biomechanopharmacology [23], with a focus on both regulation of SS and protection of ECs. It is expected to translate the key focus of Chinese medicine by integrated biomechanical concept of hemorheology and pharmacology.

\section{Other styles in Chinese medicine and biome- chanical effects}

In Chinese medical practice, acupuncture and massage also have underlining links with blood SS. At first glance, acupuncture and massage are externally applied forces to certain points or areas, but there is evidence showing that they do induce bio-responses, including regulation of blood SS. Thus, it has been reported that when needling sensation (De-Qi) was achieved, there was a local increase in blood flow at the acupuncture points. This may be one of the mechanisms accounting for the meridian system responses during acupuncture [24]. With the help of multidirectional transcranial ultrasound monitoring, it is now possible to objectively determine the reproducible effects of needle acupuncture and modern type of laser-needle acupuncture stimulation on cerebral blood flow velocity at the acupuncture targeted organ (brain) [25]. In the case of massage, dynamic changes of tissue pressure may lead to an increase in capillary flow rate and a decrease in blood apparent viscosity [26]. All of the above-mentioned changes in blood flow caused by either acupuncture or massage would elevate the level of blood SS.

The sensing mechanism of shear stress by ECs is under exploration. There is experimental evidence that a heparan sulfate component of the EC glycocalyx participates in mechanosensing that mediates NO production in response to shear stress $[27,28]$. Caveolae have been shown to be an essential component of the pathway for EC signaling asso- ciated with abrupt reduction of shear stress [29]. It was reported integrins are essential for the mechanical activation of Flk-1 by SS but not for the chemical activation of Flk-1 by VEGF [6]. The signaling events induced by SS and VEGF converge at the membrane receptor Flk-1 and that these stimuli share the Flk-1/Cbl/Akt pathway in activating IKK activation [7]. However, SS and VEGF diverge at the receptor Flk-1 in terms of the recruitment of the adapter protein Nck, and they employ different components of the complex signaling network in regulating downstream molecules, e.g., ERK and JNK [8]. It was recently reported that P2X4-mediated shear stress mechano-transduction plays an important role in vascular homeostasis, including the control of blood pressure and vascular remodeling [30]. A recent paper demonstrates that CXCR1 and CXCR2 are novel mechano-sensors mediating laminar shear stress-induced EC migration [31]. How sensory mechanisms convey information about shear stress and how is this information integrated remains insufficiently understood. These findings indicate that ligands locally generate different shear-induced responses in endothelium depending on how the force is delivered [32]. Before the emergence of a clear picture of shear-induced mechanisms of bio-responses, we can state, as an overall understanding, that ECs recognize changes in shear stress and transmit the signals to the interior of the cell to lead to cellular responses that involve changes at the levels of gene and protein expressions, and consequently cell functions (see Figure 1). Although accurate measurements of velocity profiles and SS in arteries are important, there is still no easy means to obtain such information clinically. However, the new development of non-invasive determination of SS based on ultrasound techniques is promising [33]. Measurement of SS will be an essential requirement for future research on herbs of ABCRBS, and also likely for the diagnosis of blood stasis syndrome.

Hence, we should be aware that the intensity of exercise, in conjunction with the type and duration of exercise, may result in different "pharmacological" effects. Huatuo, the great ancient surgeon of the second century, not only created five mimic-animal boxing as a modest physical exercise, but also realized the importance of blood flow as "Diseases are prevented while blood flow is promoted" [34]. An early example is the heritage drawing (see Figure 1) of physical and breathing exercises unearthed from Mawangdui Han Tombs [35]. The vivid exercising figures remind the admirers today that exercises have been an indispensable part of healthcare for our ancestors since thousands of years ago. A biological picture of blood circulation accelerated by exercise would immediately arise in the mind of any researcher in basic medical sciences: shear stress of flowing blood, the friction force between the flowing blood and EC surface, is elevated by the enhanced blood stream. This is certainly a classic annotation for the therapeutic principle of ABCRBS in traditional Chinese medicine, although of course it was not possible for Huatuo to give a definite 
elaboration of the biological effects of SS.

We believe the important issue is how to control the level of SS for healthcare. Since the increased blood flow induced by exercise affects endothelial function, we suggest that exercise, a readily self-controllable, pharmacological measure with effects of activating blood circulation and/or regulating vessel tension, could be considered as the feasible easy choice to control the level of SS [23].

\section{Preventive medicine based on blood flowing and prospect}

Chinese ancestors were aware that the lifestyle, intake of healthy food, and application of appropriate herbal medicine were all important to the maintenance of health. One of the sacred principles underlying Chinese medicine and healthcare is "preventing disease is far superior to treating a disorder". This principle is entrenched in the ancient Chinese philosophy of healthcare. The mythical Yellow Emperor said: "The sages do not treat a formed disease, but treat an unformed disease; they do not treat a formed disorder, but treat an unformed disorder. That is just so. If medicines are only used after a disease has formed, or treatments are only taken after a disorder has formed, this condition is just like digging a well when one is thirsty, or like casting knives when one is about to fight. It is so late, is it not?" [36]. Our study [37] has shown that swimming together with extracts from Salviae miltiorrhizae and Andrographis paniculata can prevent atherosclerosis, highlighting the promising biomechenopharmacological effects of combining herbal medicine with the elevation of blood SS promoted by swimming for the prevention of atherosclerosis.

To sum these up as shown in Figure 1, the combination of pharmacology with biomechanics and the practice of exercise in healthcare will provide enormous potential for achieving distinct preventive and therapeutic goals, especially by combining different sets of medicines with controlled levels of SS. Exercise needs to be well tailored for controlling SS of blood flow. To benefit from biomechanophymacology, we have to decide on the following issues: Should we go with the biomechanopharmacologically tailored exercise for controlling SS? Should we take selected diet and medicines available for protection of endothelial functions? Or should we do both? Hopefully, the disease prevention would not be an unrealistic dream.

We deeply appreciate the valuable advice and suggestions by Prof. Shu Chien. This work was supported by the National Natural Science Foundation of China (90209055, 10672192 and 90709054).

1 Pennisi E. How did cooperative behavior evolve. Science, 2005, 309: 93-93

2 Copley A L. The history of clinical hemorheology. Clin Hemorheol, 1985, 5: 765-811

3 Muller S, Labrador V, Da Isla N, et al. From hemorheology to vascular mechanobiology: An overview. Clin Hemorheol Microcirc, 2004, 30: 185-200

4 Chiu J-J, Chien S. Effects of disturbed flow on vascular endothelium: Pathophysiological basis and clinical perspectives. Physiol Rev, 2011, 91: 327-387

5 Krizanac-Bengez L, Mayberg M R, Janigro D. The cerebral vasculature as a therapeutic target for neurological disorders and the role of shear stress in vascular homeostatis and pathophysiology. Neurol Res, 2004, 26: 846-853

6 Wang Y X, Miao H, Li S, et al. Interplay between integrins and FLK-1 in shear stress-induced signaling. Am J Physiol Cell Physiol, 2002, 283: C1540-C1547

7 Wang Y X, Chang J, Li Y C, et al. Shear stress and VEGF activate IKK via the Flk-1/Cbl/Akt signaling pathway. Am J Physiol Heart Circ Physiol, 2004, 286: H685-H692

8 Wang Y, Chang J, Chen K D, et al. Selective adapter recruitment and differential signaling networks by VEGF vs. shear stress. Proc Natl Acad Sci USA, 2007, 104: 8875-8879

9 Wang J, Chen K J, Weng W L, et al. Research on diagnostic criteria of blood-stasis symptom-complex (in Chinese). J Integrated Trad Chin West Med, 1988, 8: 585-589

10 Liao F L, Han D, Ouyang Z G, et al. The influences of Ligustrazine and shear stress on PS transformation of endothelial cell membrane investigated by evanescent wave exciting (in Chinese). Acta Biophys Sin, 2003, 19: 92-96

11 Xie L X, Durairajan S S K, Lu J H, et al. The effect of salvianolic acid $\mathrm{B}$ combined with laminar shear stress on TNF-alpha-stimulated adhesion molecule expression in human aortic endothelial cells. Clin Hemorheol Microcirc, 2010, 44: 245-258

12 O’Brien J R. Shear-induced platelet aggregation. Lancet, 1990. 335: 711-713

13 Liao F L, Li B. Inhibition of shear-induced platelet aggregation by Chinese herbal medicines. Clin Hemorheol Microcirc, 1997, 17: 315-318

14 Liao F L, Jiao L G. Ligustrazini, allicin and shear-induced platelet aggregation. Clin Hemorheol Microcirc, 2000, 22: 167-168

15 Li M, Handa S, Ikeda Y, et al. Specific inhibiting characteristics of tetramethylpyrazine, one of the active ingredients of the Chinese herbal medicine 'chuanxiong', on platelet thrombus formation under high shear rates. Thromb Res, 2001, 104: 15-28

16 Li M, Zhao C, Wong R N S, et al. Inhibition of shear-induced platelet aggregation in rat by tetramethylpyrazine and salvianolic acid B. Clin Hemorheol Microcirc, 2004, 31: 97-103

17 Ma W, Sun Y, Han D, et al. Cytoskeletal response of microvessel endothelial cells to an applied stress force at the submicrometer scale studied by atomic force microscopy. Micros Res Tech, 2006, 69: 784-793

18 Han J Y, Fan J Y, Horie Y, et al. Ameliorating effects of compounds derived from Salvia miltiorrhiza root extract on microcirculatory disturbance and target organ injury by ischemia and reperfusion. Pharmacol Ther, 2008, 117: 280-295

19 Liao F L, Peng J, Li W. Microthrombogenesis induced by rose Bengal under ordinary microscopic illumination. In: Messmer K, Kuble W M, eds. Sixth World Congress for Microcirculation, 1996 Aug 25-30, Munich. Monduzzi Editore, 1996. 479-482

20 Chen K J, Shi Z X, Practical Works of Blood Stasis Syndrome (in Chinese). Beijing: The People's Health Publisher,1999

21 Lu X Y, Shi D Z, Xu H, et al. Clinical study on effect of xiongshao capsule on restenosis after percutaneous coronary intervention (in Chinese). Chin J Integrated Trad Chin West Med, 2006, 26: 13-17

$22 \mathrm{Xu} \mathrm{H}$, Shi D Z, Chen K J, et al. Clinical effect of xiongshao capsule on preventing restenosis post-PTCA or/and Stenting (in Chinese). Chin J Integrated Trad Chin West Med, 2000, 20: 494-497

23 Liao F L, Li M, Han D, et al. Biomechanopharmacology: A new borderline discipline. Trends Pharmaco Sci, 2006, 27: 287-289

24 Kuo T C, Lin C W, Ho F M. The soreness and numbness effect of acupuncture on skin blood flow. Am J Chin Med, 2004, 32: 117-129

25 Litscher G. Bioengineering assessment of acupuncture, Part 3: Ultrasound. Crit Rev Biomed Eng, 2006, 34: 295-325 
26 Liu Y F, Xu S X, Yan J T, et al. Capillary blood flow with dynamical change of tissue pressure caused by exterior force (in Chinese). J Biomed Eng, 2004, 21: 699-703

27 Florian J A, Kosky J R, Ainslie K, et al. Heparan sulfate proteoglycan is a mechanosensor on endothelial cells. Circ Res, 2003, 93: E136-E142

28 Tarbell J M, Weinbaum S, Kamm R D. Cellular fluid mechanics and mechanotransduction. Ann Biomed Eng, 2005, 33: 1719-1723

29 Milovanova T, Chatterjee S, Hawkins B J, et al. Caveolae are an essential component of the pathway for endothelial cell signaling associated with abrupt reduction of shear stress. BBA-Mol Cell Res, 2008, 1783: 1866-1875

30 Yamamoto K, Ando J. Blood flow sensing mechanism via calcium signaling in vascular endothelium (in Japanese). Yakugaku Zasshi, 2010, 130: 1407-1411

31 Zeng Y, Sun H R, Yu C, et al. CXCR1 and CXCR2 are novel mechano-sensors mediating laminar shear stress-induced endothelial cell migration. Cytokine, 2011, 53: 42-51

32 Chretien M L, Zhang M, Jackson M R, et al. Mechanotransduction by endothelial cells is locally generated, direction-dependent, and ligand-specific. J Cell Physiol, 2010, 224: 352-361

33 Remuzzi A, Ene-Iordache B, Mosconi L, et al. Radial artery wall shear stress evaluation in patients with arteriovenous fistula for hemodialysis access. Biorheology, 2003, 40: 423-430

34 Li J W. Brilliant Achievemenst in Chinese Medicine (in Chinese). Beijing: Chinese Medicine Press of China, 1998

35 He Z J, Wu H Z. Photographic Collection of Chinese Medical Relics (in Chinese). Chengdu: Sichuan People's Publisher, 2001

36 Zhu M. The Medical Classic of the Yellow Emperor (in Chinese). Beijing: Foreign Language Press, 2001

37 You Y, Liu W, Li Y, et al. Joint preventive effects of swimming and Shenlian extract on rat atherosclerosis. Clin Hemorheol Microcirc, 2011, 47: 187-198

Open Access This article is distributed under the terms of the Creative Commons Attribution License which permits any use, distribution, and reproduction in any medium, provided the original author(s) and source are credited 